Revue européenne des sciences sociales

European Journal of Social Sciences

XL-124 | 2002

Histoire, philosophie et sociologie des sciences

\title{
Le balancier sociologique français : entre individus et structures
}

Charles-Henry Cuin

\section{OpenEdition}

Journals

Édition électronique

URL : http://journals.openedition.org/ress/591

DOI : 10.4000/ress.591

ISSN : 1663-4446

Éditeur

Librairie Droz

Édition imprimée

Date de publication : 1 août 2002

Pagination : 253-262

ISBN : 2-600-00806-3

ISSN : 0048-8046

Référence électronique

Charles-Henry Cuin, "Le balancier sociologique français : entre individus et structures », Revue européenne des sciences sociales [En ligne], XL-124 | 2002, mis en ligne le 01 décembre 2009, consulté le 01 mai 2019. URL : http://journals.openedition.org/ress/591 ; DOl : 10.4000/ress.591 


\section{LE BALANCIER SOCIOLOGIQUE FRANÇAIS : ENTRE INDIVIDUS ET STRUCTURES}

I - En 1973, au plus fort du débat français sur l'École et les inégalités (Bourdieu et Passeron, 1970; Baudelot et Establet, 1971), Raymond Boudon publiait un ouvrage qui n'apportait pas seulement une contribution décisive à la sociologie de la scolarisation et de la mobilité sociale mais qui constituait aussi un événement théorique peut-être sans précédent dans l'histoire de la sociologie française. L'Inégalité des chances entreprenait en effet d'analyser un phénomène macrosocial «la mobilité sociale dans les sociétés industrielles», sous-titre du livre - comme le résultat d'un processus complexe conjuguant structures sociales et conduites individuelles (Boudon, 1973). Si, rétrospectivement, l'exercice peut paraître naturel, voire banal, il faut se souvenir qu'il intervenait dans un contexte paradigmatique où les déterminismes structurels sévissaient et où, dans les meilleurs cas, les conduites individuelles étaient réduites à l'actualisation de ces déterminismes. La sociologie française commençait tout juste à sortir d'un bref mais pénible cauchemar dogmatique où les vieux démons marxistes avaient conclu un étrange pacte avec de jeunes preux structuralistes.

Tel un balancier, la sociologie française était en train de quitter un de ses pôles pour reprendre une nouvelle course vers le pôle opposé, qu'elle ne tarderait d'ailleurs pas à atteindre prochainement, au cours des années quatre-vingt-dix. L'originalité de l'entreprise boudonienne ne tenait pas seulement au fait qu'elle avait anticipé ce mouvement mais, surtout, à ce qu'elle cherchait à l'arrêter avant qu'il ne conduise à nouveau vers une position extrême où ce ne seraient plus les structures qui tiendraient désormais le devant de la scène sociologique, mais les seuls individus. Dans l'ouvrage de 1973, comme dans quelques rares autres (e.g. Crozier et Friedberg, 1977), les protagonistes du drame social étaient de deux types - les structures et les individus - sans qu'aucun l'emporte sur l'autre, les unes et les uns n'existant que par les autres et leur jeu animant la scène d'événements aussi divers que, parfois, inattendus (Boudon, 1977).

Cette oscillation marque toute l'histoire de la sociologie, en particulier dans le cas français. Dès les premiers balbutiements de la discipline avec la théorie mimétique de Tarde (1890/1979), c'est l'individualisme qui s'impose comme principe théorique et méthodologique d'analyse du social. Par réaction, dans ses textes doctrinaux, Durkheim (1893/1991 et 1895/1987) renverra le balancier dans l'autre direction, celle des déterminismes structurels tout en pratiquant souvent (Durkheim, 1897/1960), de façon parallèle, un individualisme méthodologique parfaitement hérétique (Cuin, 1997). Lorsque, au sortir de la Seconde Guerre mondiale, la sociologie française se reconstruisit sur les décombres depuis longtemps éteints du durkheimisme, c'est au retour à une forme d'individualisme que 
l'on en appela de tous côtés ou presque. Tandis que les philosophes - existentialistes ou personnalistes - proclamaient farouchement que les faits sociaux n'étaient pas des «choses» et que les individus étaient des sujets, que Stoetzel exhortait à «oublier Durkheim», on n'avait plus d'yeux que pour la «sociologie analytique» (et son outil favori, l'enquête par questionnaires) que des psychosociologues américains comme Stouffer et Lazarsfeld avaient réussi à imposer comme seule et unique voie de connaissance et d'explication d'une réalité sociale réduite aux comportements individuels (Cuin et Gresle, 1992). Revenu du côté des individus, le balancier se préparait pourtant, sous la double influence du structuralisme, qui faisait alors florès en linguistique et en anthropologie, et d'un marxisme radicalisé, à regagner brusquement le pôle opposé, ressuscitant une «philosophie sans sujet» (Bourdieu et Passeron, 1967).

La sociologie poursuit ainsi une course pendulaire qui la ramène avec insistance soit vers les individus soit vers les structures, ne faisant généralement pas de longue halte dans une position intermédiaire où elle réussit pourtant ses meilleures performances.

II - Le balancier se situe actuellement vers le pôle individualiste. Ce n'est pas seulement attesté par la nature des paradigmes aujourd'hui dominants. Ça l'est aussi, et de façon évidemment liée, par le développement d'une sensibilité idéologique et culturelle largement favorable à une représentation individualiste de la réalité sociale.

En France, le succès, depuis la fin des années soixante-dix, du mot d'ordre de «retour de l'acteur» (Touraine, 1984) a correspondu à l'épuisement heuristique des divers avatars de la pensée structuraliste. Celle-ci avait atteint, avec le structuralo-marxisme d'inspiration althusserienne, au comble du déterminisme et du systématisme abstrait (Althusser, 1966; Poulantzas, 1970). Pour avoir chassé l'action de la réalité socio-historique, ce paradigme se révélait en effet incapable d'expliquer quoi que ce soit. Le social se retrouvait réduit à un épiphénomène de l'économique, voire du politique, et des problématiques entières de la sociologie étaient soit frappées d'invalidité soit dénoncées comme idéologiquement perverses. Ainsi, dans un domaine que nous connaissons un peu (Cuin, 1993) et qui était alors l'un des plus développés de la discipline, les phénomènes de mobilité sociale furent considérés comme des illusions d'optique engendrées par la seule évolution des rapports sociaux de type capitaliste. Ce n'étaient pas les individus qui se mouvaient mais les structures qui se reproduisaient (Poulantzas, 1974). Peu importait donc avec qui cette reproduction s'exécutait et de qui elle avait besoin puisque, par la grâce d'un providentiel mécanisme de régulation, le système social était en mesure de transmuter les individus concernés en agents conformes et dociles (Baudelot et Establet, 1971).

La réaction individualiste fut saine à tous égards. On montra d'abord que, dans les organisations les plus structurées, les individus savaient et pouvaient devenir des acteurs (Crozier et Friedberg, 1977); que la structure scolaire de nos société était en grande partie le produit de décisions et de conduites individuelles (Boudon, 1973); plus généralement, que les phénomènes historiques étaient provoqués par des acteurs qui, avant de devenir collectifs, étaient d'abord des individus en lutte pour leur identité socio-culturelle (Touraine, 1973). En dépit de la résistance d'un courant farouchement attaché à un déterminisme structurel réduisant l'acteur 
à un agent (Bourdieu, 1980), le balancier se dirigeait ainsi vers une position intermédiaire où la sociologie pourrait retrouver la plénitude de ses compétences.

Pourtant, le «retour de l'acteur» fut vite dépassé par celui du «sujet», et le balancier poursuivit sa course, comme mu par une force irrésistible. Dans cette course, les structures s'effacèrent de plus en plus du paysage théorique - jusqu'à en disparaître complètement dans certains cas. Le «choix rationnel » qui constituait un modèle efficace d'interprétation des conduites des acteurs dans des systèmes structurés, tendit à se naturaliser et, partant, à perdre une grande partie de ses capacités heuristiques. On s'intéressa moins à ce que la rationalité des acteurs et de leurs conduites devait aux situations sociales et aux contraintes structurelles et davantage à ce qu'elle devait à la cognition humaine (Boudon, 1990), voire à la nature physiologique de l'homme (Sperber, 1996). Dans d'autres perspectives, on tenta de dégager l'action de sa gangue culturelle et même contextuelle, pour atteindre le noyau dur (pur?) de la subjectivité intrinsèque, celle par laquelle l'acteur ne serait plus qu'un «sujet» plus ou moins largement délié des contraintes normatives et/ou de la quête de ses seules «utilités» individuelles. La théorie et l'analyse sociologiques y trouvèrent assurément bénéfice, en particulier en montrant que l'action ne pouvait se réduire ni à de l'intégration ni à de la stratégie et que le processus de la «subjectivation» permettait d'enrichir la compréhension des représentations et des conduites (Dubet, 1994).

Enfin, inspirés par l'interactionnisme anglo-saxon (e.g. Blumer, 1969; Strauss, 1978), d'autres courants choisirent de dissoudre le social dans les interactions individuelles qui le constituent et l'expriment, considérant ainsi ses structures comme des équilibres essentiellement précaires et conjoncturels, intervenant dans l'action sociale moins comme des données objectives et contraignantes que comme des situations toujours négociables. Dès lors, l'action et les acteurs devenaient les seuls principes d'analyse d'une réalité sociale réduite au produit momentané de cette négociation (Pharo, 1993), voire une pure et simple construction subjective symboliquement partagée (Maffesoli, 1985). Dans ce dernier cas, la société n'était plus qu'une représentation dont l'analyse pouvait, pour d'aucuns, se résoudre dans la description qu'en faisaient ses acteurs (Fornel et al., 2001).

Que la sociologie y ait conquis des concepts utiles et des champs d'investigation considérables est indéniable. Que la notion, jusque là bien floue, de «structure(s) sociales(s)» s'y soit très avantageusement assouplie et enrichie ne l'est pas moins. Sans doute aussi la discipline y gagna-t-elle un savoir supérieur en matière de psychologie humaine (e.g. Ehrenberg, 1998). Au-delà de l'homo sociologicus et de l'homo economicus de ses analyses classiques, la sociologie devenait, à l'instar de la psychologie, experte en humanité - même si, ce faisant, elle rejetait purement et simplement les acquis les plus solides de la théorie de l'inconscient qui ne cesse de rappeler qu'il y a loin de l'acte à parole (Cuin, 2000a).

III - Il serait risqué et, sans doute, largement gratuit d'engager une interprétation sociologique des périodes du balancement sociologique entre individus et structures. L'étude des rapports entre science et société nourrit toute une littérature dont le degré de pertinence est inversement proportionnel à celui de la «dureté» des sciences considérées. Dans le cas des sciences sociales, ces rapports sont généralement trop évidents pour ne pas être suspects. En outre, leur analyse rencontre fatalement l'aporie consistant à expliquer des orientations sociologiques que leurs 
défenseurs expliquent eux-mêmes par des raisons sociologiques. Ainsi, les sociologies du «sujet» sont explicitement fondées sur la thèse de «l'émergence» historique de nouveaux types d'actions et d'acteurs dans nos sociétés (Dubet, 1994; Lahire, 1998). Leur validité théorique étant intrinsèquement liée à leur pertinence socio-historique, il est difficile de ne pas reconnaître l'une sans partager l'autre. On se limitera donc ici à quelques observations éparses.

D'une part, on constatera qu'il existe une forte demande sociale concernant la «compréhension» du social entendue comme une intelligibilité quasi immédiate du concret, par opposition à celle qui recourt à des explications hypothétiques et abstraites. À cette fin, l'une des procédures les plus efficaces et les moins coûteuses intellectuellement consiste à découvrir et à exhiber le sens que les acteurs sociaux donnent à ce qu'ils font, ces discours étant censés contenir l'essentiel de l'explication des conduites et, à terme, du social. À rebours de la méthode weberienne (dont la sociologie contemporaine se réclame pourtant bruyamment) qui n'exploite la subjectivité des acteurs que pour construire des idéaux-types puis des hypothèses scientifiquement testables, cette «compréhension» recherche dans les matériaux empiriques - discursifs ou objectifs - des significations dont la véracité n'est souvent fondée que sur l'empathie ou l'expérience subjective de l'observateur et, à travers lui, du lecteur (Quéré, 1999). On pourrait ici, à titre de conjecture, avancer que l'intelligibilité que l'on attend des sciences sociales est plus de type descriptif, voire phénoménologique, que de type explicatif. C'est la raison pour laquelle l'interprétation, voire l'herméneutique, y rencontrent plus de succès que la démonstration (Cuin, 2000).

D'où l'engouement pour les entretiens approfondis (e.g., Lahire, 2002), les observations (si possible «participantes») fouillées, pénétrant au plus loin de l'intimité, sondant les cœurs et les armoires (e.g., Kaufmann, 1992), toutes entreprises dans lesquelles les méthodes de l'anthropologie font merveille. D'où le succès public de la sociologie de la vie quotidienne, de l'ethnologie des institutions, de l'anthropologie des comportements, de tous ces «romans vrais» que nous attendons que l'on nous donne sur nous-mêmes ou sur les autres. Si leur lecture nous rend moins ignorants, il est moins sûr que nous en sortions plus savants.

D'autre part, on peut observer que les objets que l'on propose aux sociologues sont souvent, et de plus en plus, non des phénomènes sociaux mais des artefacts statistiques. Il est alors naturel que, pour expliquer la taille ou la variation d'un taux (de divorce, de délinquance, de consommation quelconque, etc.), les sociologues se bornent - car c'est souvent suffisant - à montrer pourquoi il y a (de plus en) plus ou (de moins en) moins d'individus qui pensent ou ressentent la même chose. Mais les artefacts statistiques ne sont pas des phénomènes sociaux émergents. La survenue d'une grève n'est pas fonction du nombre de grévistes, celle d'une mode vestimentaire ne se mesure pas à la proportion des gens qui la suivent. Ainsi, si l'on connaît de mieux en mieux pourquoi les gens font (pensent, croient) ce qu'ils font (pensent, croient), on ne connaît pas nécessairement mieux ce qu'ils font collectivement. Les bonnes raisons (Boudon, 1992) qu'ont les grévistes de participer à une grève ne suffisent pas à expliquer cette grève. Bref, et pour garder ces exemples, ce n'est pas parce que l'on comprend (et de mieux en mieux) pourquoi des gens font une grève ou suivent une mode que l'on comprend mieux pourquoi cette grève éclate ou cette mode s'impose. 
IV - Une analyse «interne» du phénomène est sans doute plus éclairante encore.

À l'encontre de ce qu'on a cru pouvoir établir (Alexander et al., 1987), le tournant micrologique pris par la sociologie en général et la sociologie française en particulier n'explique que très partiellement cette négligence des structures. Les approches macroscopiques ne sont pas nécessairement de type «holiste». Elles peuvent en effet, tout aussi bien et souvent mieux, utiliser une méthodologie individualiste considérant les phénomènes macro-sociaux comme résultant de l'agrégation compositive de conduites individuelles (Boudon, 1979; Coleman, 1990). Quant aux approches micrologiques, si elles accordent naturellement une importance essentielle aux conduites individuelles, les plus pertinentes d'entre elles ne les prennent en considération que comme intervenant dans des situations, des configurations ou autres systèmes structurés (Elias, 1987/1991; Goffman, 1974/1991).

La prévalence des analyses ignoreuses des structures vient plutôt de deux tendances fortes du champ théorique actuel. Il s'agit, d'une part, du succès rencontré par les sociologies du «sujet». Après avoir longtemps été réservé aux seules entreprises psychologique et anthropologique, l'individu s'est aujourd'hui imposé comme un objet sociologique légitime et central. Plus exactement, ce sont les dimensions non socialisées (au sens le plus déterministe du terme) ou encore non rationnelles de l'individu et de ses conduites qui ont été intégrées dans le champ sociologique. Alors qu'une psychologie de convention constituée par quelques postulats plus ou moins déterministes relatifs à la nature sociale de l'acteur suffisait à fonder des schémas explicatifs satisfaisants pour l'analyse sociologique de l'action, de ses causes et de ses conséquences, on s'est de plus en plus intéressé à la subjectivité individuelle comme construction sociale. À côté de l'individu socialisé conformant ses conduites à des normes et reproduisant ainsi des modèles structurels, à côté également de l'individu calculateur cherchant à optimiser ses gains, on s'est interrogé sur les conditions sociales dans lesquelles l'individu pouvait être aussi un authentique sujet - c'est-à-dire un acteur intentionnel, innovant, voire créatif (Joas, 1992/1999). Plus généralement, les sociologues consacrent une grande part de leurs recherches à la définition de modèles d'action individuelle qui, évidemment, se révèlent toujours insatisfaisants dans la mesure où ils ne parviennent jamais à rendre compte de l'intégralité des conduites collectives. On esquissera plus bas quelques réflexions sur le caractère nécessairement aporétique d'une telle entreprise.

Mais la raison sans doute la plus importante de l'hyperindividualisme de la sociologie contemporaine est à rechercher dans une conception qui, outrepassant le caractère purement méthodologique de la démarche individualiste, tend à assimiler phénomènes sociaux et conduites collectives. Arguant - avec raison - de ce que la réalité sociale n'est que de l'action objectivée, on est enclin à remonter plus ou moins directement des conduites individuelles aux phénomènes collectifs qu'elles engendrent. On est donc conduit à estimer que l'explication sociologique peut, pour l'essentiel, être réduite à celle de ces conduites - et, le plus souvent, à la mise en évidence des raisons subjectives de l'action individuelles. C'est là, évidemment, faire bon marché de cette phase essentielle de la méthode individualiste qui réclame d'identifier précisément les processus, souvent formidablement complexes, selon lesquels les conduites s'agrègent et se composent. De fait, même lorsque cette agrégation est de type additif (ce qui n'est stricte- 
ment le cas que lorsque le phénomène social considéré n'est qu'un artefact statistique: une proportion, un taux), le phénomène émergeant n'échappe qu'exceptionnellement à tout effet structurel. Même lorsque les acteurs sont entièrement libres (culturellement, socialement, économiquement) de choisir une conduite, leur choix se trouve généralement limité par des contraintes incontournables: même si tout le monde décide de circuler à vélo, le taux de cyclistes ne dépassera jamais le nombre de vélos disponibles. Il n'y a que dans le domaine des représentations et de certaines conduites ne nécessitant pas des instruments étroitement spécifiés, que les acteurs échappent à ces contraintes objectives: la croyance dans le surnaturel ou le recours au suicide peuvent affecter $100 \%$ d'une population.

Ainsi, pour reprendre un exemple cher à R. Boudon, l'explication de la file d'attente qui se forme le dimanche matin devant la pâtisserie du quartier ne requiert pas seulement la connaissance des raisons de chalands ayant tous décidé d'acheter des gâteaux au même moment mais aussi celle du nombre des employés de la boutique et de l'organisation de leur travail.

À cela, le «subjectivisme» sociologique se fera fort de rétorquer que les acteurs intègrent les contraintes structurelles dans leur définition de la situation et, donc, de leurs conduites, ou encore qu'il est important de savoir pourquoi et dans quelle mesure ils les acceptent et, dans certains cas, peuvent les susciter. Il ne restera pas moins vrai qu'ils ne connaissent et, a fortiori, ne peuvent intégrer généralement toutes ces contraintes et que, de ce fait et quoi qu'on en dise (Giddens, 1984/1987), le rôle des sociologues est indispensable à l'explication sociologique (Cuin, 2000).

V - Tel un balancier, la sociologie va donc régulièrement d'une extrémité à l'autre de cette course qui la ramène avec insistance soit vers les individus soit vers les structures. Ce n'est que lorsqu'elle s'est heurtée aux limites de l'une de ces alternatives qu'elle rebrousse chemin dans la direction de l'autre. Mais elle s'arrête rarement en cours de route, dans les parages de ce qui, pour un mouvement pendulaire, devrait être une halte naturelle et définitive - à mi-chemin entre individualisme et structuralisme.

Dans le cas de la pâtisserie de tout à l'heure, les sociologues sont généralement tentés de ne choisir qu'une seule alternative: rester dehors pour interviewer les clients ou entrer pour étudier la nature du service. Ce n'est que plus rarement qu'ils avisent que la file d'attente résulte à la fois de l'agrégation des gourmandises individuelles et du malthusianisme salarial et/ou de l'impéritie du patron que ladite file d'attente, si gourmands que soient les clients, n'existerait pas si le nombre des vendeuses était décuplé et/ou leurs tâches mieux coordonnées. Analytiquement, il ne saurait donc y avoir aucune primauté - ni ontologique ni génétique - d'une des deux dimensions sur l'autre. Dans un phénomène macro-social, les structures n'existent qu'en relation avec les conduites individuelles et celles-ci qu'en relation avec celles-là.

Pourtant, dans ce domaine, les conceptions théoriques sont relativement peu efficaces pour la solution des questions pratiques qui se posent à l'explication sociologique. Il ne suffit pas d'invoquer la «dualité du structurel» (Giddens, op. cit.) ou encore l' «interdépendance » (Elias, 1970/1991) pour percer le mystère des processus générateurs par lesquels les conduites engendrent des phénomènes 
sociaux et ceux par lesquels les «structures» ainsi engendrées vont s'imposer à ces conduites et à leurs acteurs. La solution de la «fusion centrale» (Archer, 1995) entre individus et structures n'est satisfaisante qu'au plan théorique. Affirmer qu'il faut communier aux deux espèces dans une même analyse ne dit rien de la façon dont il faut mener l'analyse. Trop souvent, même, la représentation de la réalité sociale comme le produit indissociable de conduites et de structures peut inviter à choisir indifféremment l'une ou l'autre de ces entrées. Si les structures ne sont que des conduites objectivées et les conduites des effets de structures, on estime alors pouvoir atteindre les unes par les autres, l'approche par les structures permettant de déduire les conduites et vice-versa. Mais, dans tous ces cas, l'analyse ne peut se passer du recours à un principe de transmutation des unes en les autres - ainsi le concept d'habitus qui permet d'expliquer que, parce qu'elles sont structurellement engendrées, des conduites individuelles puissent présenter des régularités au niveau collectif.

Ce qui a fait l'originalité et la fécondité de la solution adoptée par R. Boudon dans L'Inégalité des chances est le double refus d'accéder à l'explication des phénomènes étudiés soit par les seuls individus soit par les seules structures. Renvoyant dos à dos l'individualisme des théories anglo-saxonnes de l'époque (Blau et Duncan, 1967; Jenks, 1972/1983) et le structuralisme plus ou moins "génétique» de ses collègues français, la démarche boudonienne construit la mobilité sociale comme un processus de «distribution sociale», grâce auquel il devient possible de spécifier les multiples interactions qui se jouent entre individus et structures sociales. Cette théorie est trop connue pour ne pas pouvoir être grossièrement résumée: l'allocation des positions sociales aux individus dépend, d'une part, de la répartition des positions statutaires dans la structure socioprofessionnelle et, d'autre part, de la répartition d'un certain nombre de caractéristiques individuelles (principalement: origine sociale et niveau d'instruction) dans la population; c'est la non-congruence existant entre ces répartitions qui, pour l'essentiel, est génératrice du phénomène de la mobilité sociale. Ainsi, il apparaît bien, d'une part, que les décisions individuelles (en particulier en matière scolaire) sont à la fois largement affectées par l'état momentané de la structure sociale à laquelle les individus appartiennent et de la «structure scolaire» qu'ils cherchent à intégrer et, d'autre part, que les conduites résultant de ces décisions participent à la définition de ces structures. Mais l'extrême complexité de ces interactions interdit tout à la fois de considérer les individus comme les seuls «acteurs» de leur carrière (scolaire et sociale) et de considérer les structures sociales comme déterminant seules les conduites de ces derniers. Dans la mesure où les individus définissent ces conduites en référence à leur perception de la «valeur d'échange» des diplômes sur le marché de l'allocation statutaire, leurs décisions influencent également l'équilibre de ce marché et, de ce fait, le degré de congruence entre «structure scolaire» et structure sociale - ce degré de congruence jouant un rôle déterminant dans la génération de la mobilité sociale.

Ainsi, ni l'analyse de la subjectivité des acteurs ni la mise au jours de logiques structurelles, voire institutionnelles, n'auraient pu s'avérer capables, même conjuguées, de fournir d'explication satisfaisante à un grand nombre d'observations empiriques relatives à la mobilité sociale et à l'inégalité des chances dans les sociétés industrielles. À l'évidence, il était nécessaire de recourir, via la modélisation, à une analyse en terme de processus. 
VI - Sans doute faut-il reconnaître que, au cours de la période «individualiste » que la sociologie vient de traverser, l'enrichissement corrélatif de son savoir sur l'acteur et l'action s'est accompagné d'un appauvrissement relatif du savoir sur le social. Tout s'est un peu passé comme si les progrès accomplis dans le domaine de la sociologie de l'action se firent aux dépens de la sociologie tout court. Pour avoir réduit l'analyse sociologique à celle de l'action et de l'acteur, voire du sujet, la sociologie a accumulé de plus en plus de savoir sur l'acteur et de moins en moins sur la réalité sociale.

$\mathrm{Si}$, comme Weber nous l'a répété et nous en a finalement convaincus, les phénomènes sociaux ne sont que de l'action objectivée (Weber, 1922/1971), il n'est pas moins vrai que le problème de l'analyse sociologique est autant celui de l'élucidation des processus de cette objectivation (ou, en termes plus modernes, des processus de composition d'où émergent les phénomènes sociaux) que celui de l'interprétation de l'action. Les sociologies de l'action, comme celles de l'acteur ou du sujet, ne sont ainsi que des sociologies d'objets spécifiques; elles ne sont ni toute la sociologie ni, moins encore, l'alpha et l'oméga de l'analyse des phénomènes collectifs.

S'il est vrai que les individus agissent plus ou moins librement et que leurs actions déterminent des «configurations » (Elias, 1970/1991) qui influencent à leur tour leurs conduites, il est également vrai que la temporalité inhérente à tout processus socio-historique réclame du sociologue qu'il considère à un moment ou à un autre ces conduites et ces structures comme des données qui, bien qu'elles résultent les unes des autres, constituent à un moment donné de l'analyse des variables indépendantes. Selon le moment de l'entreprise explicative, la démarche sera soit individualiste soit structuraliste. La subjectivité des acteurs (ou les discours qui l'expriment) sera éventuellement suffisante pour expliquer ce que font ces derniers mais, dans la quasi totalité des cas, ne le sera pas pour expliquer le phénomène collectif («structurel» ou «configurationnel») émergeant. Dans certains cas extrêmes, il pourra même se faire que les structures mènent à elles seules tout le jeu: si, par exemple (Cherkaoui, 1997), dans une région donnée, les Protestants sont en nombre inférieur aux Catholiques, la proportion de mariages mixtes sera nécessairement plus importante parmi les premiers que parmi les seconds - et ce n'est certainement pas le discours des intéressés, ou des hypothèses sur leur libéralisme différentiel, qui livrera la clé du phénomène !

Si la sociologie retrouve l'ambition, qui fut celle de ses fondateurs, d'expliquer les «phénomènes sociaux» (i.e., collectifs), elle doit alors retrouver aussi le chemin d'une démarche rendant justice à l'analyse en terme de processus, voire celui d'un «réalisme méthodologique» qui ne saurait évidemment être étendu à ou déduit d'un réalisme théorique (Archer, op. cit.).

Le balancier sociologique devra sans doute reprendre sa course vers le pôle structurel pour que se présente à nouveau l'un de ces moments privilégiés où, parce que le balancier est à son point d'équilibre, la sociologie est à son meilleur. Il reste - et c'est là un réel motif d'espoir - qu'à chacun de ses voyages, le balancier acquiert une énergie nouvelle et vient enrichir notre connaissance de la réalité sociale par l'affinement des concepts centraux de l'analyse sociologique que sont ceux d'《individu» et de «structure».

Département de sociologie - LAPSAC

Université Victor Segalen - Bordeaux 2 


\section{RÉFÉRENCES BIBLIOGRAPHIQUES}

Alexander J. et al., 1987, The Micro-Macro Link, Univ. of California Press.

Althusser L., 1966, Lire le Capital, Maspero.

Archer M.S., 1995, Realist Social Theory, Cambridge Univ. Press.

Baudelot C. et Establet R., 1971, L'École capitaliste en France, Maspero.

Blau P.M. et O.D. Duncan, 1967, The American Occupational Structure, Wiley.

Blumer H., 1969, Symbolic Interactionism, Prentice Hall.

Boudon R., 1973, L'Inégalité des chances, A. Colin.

Boudon R., 1977, Effets pervers et ordre social, P.U.F.

Boudon R., 1979, La Logique du social, Hachette.

Boudon R., 1990, L'Art de se persuader, Fayard.

Boudon R. (éd.), 1992, Traité de sociologie, P.U.F.

Bourdieu P., 1980, Le Sens pratique, Minuit.

Bourdieu P. et Passeron J.-Cl., 1967, Sociology and Philosophy in France since 1945 : Death and Resurrection of a Philosophy without Subject, "Social Research", 34 (1), 162-212.

Bourdieu P. et Passeron J.-Cl., 1970, La Reproduction, Minuit.

Cherkaoui M., 1997, Le réel et ses niveaux: peut-on toujours fonder la macrologie sur la micrologie? "Revue française de sociologie", XXXVIII-3, 497-524.

Coleman J.S., 1990, Foundations of Social Theory, Belknap Press.

Fornel M., Ogien, A. et Quéré, L. (éds), 2001, L'Ethnométhodologie, une sociologie radicale, La Découverte.

Crozier M. et Friedberg E., 1977, L'Acteur et le système, Seuil.

Cuin Ch.-H., 1993, Les Sociologues et la mobilité sociale, P.U.F.

Cuin Ch.-H. (éd.), 1997, Durkheim d' un siècle à l'autre, P.U.F.

Cuin Ch.-H., 2000, Ce que (ne) font (pas) les sociologues, Droz.

Cuin Ch.-H., 2000a, Sociologie sans paroles: Durkheim et le discours des acteurs, in Borlandi M. et Cherkaoui M. (éds), Le Suicide. Un siècle après Durkheim, P.U.F., 125-146.

Cuin Ch.-H. et Gresle F., 1992, Histoire de la sociologie : 2. Depuis 1918, La Découverte.

Dubet F., 1994, Sociologie de l'expérience, Seuil.

Durkheim É., 1893/1991, De la division du travail social, P.U.F.

Durkheim É., 1895/1987, Les Règles de la méthode sociologique, P.U.F.

Durkheim É., 1897/1960, Le Suicide, P.U.F.

Ehrenberg A., 1998, La Fatigue d'être soi, Odile Jacob.

Elias N., 1970/1991, Qu'est-ce que la sociologie?, Agora.

Elias N., 1987/1991, La Société des individus, Fayard.

Giddens A., 1984/1987, La Constitution de la société, P.U.F.

Goffman E., 1974/1991, Les Cadres de l'expérience, Minuit.

Jenks C., 1972/1983, L'Inégalité, P.U.F.

Joas H., 1992/1999, La Créativité de l'agir, Cerf.

Kaufmann J.-Cl., 1992, La Trame conjugale, Nathan.

Lahire B., 1998, L'Homme pluriel, Nathan.

Lahire B., 2002, Portraits sociologiques, Nathan.

Maffesoli M., 1985, La Connaissance ordinaire, Méridiens Klincksieck.

Pharo P., 1993, Le Sens de l'action et le comportement d'autrui, L'Harmattan.

Poulantzas N., 1970, Pouvoir politique et classes sociales, Maspero.

Poulantzas N., 1974, Les Classes sociales dans le capitalisme d'aujourd' hui, Seuil. 
Quéré L., 1999, La Sociologie à l'épreuve de l'herméneutique, L'Harmattan.

Sperber D., 1996, La Contagion des idées, O. Jacob.

Strauss A., 1978, Negociations, Adline.

Tarde G., 1890/1979, Les Lois de l' imitation, Slatkine Reprints.

Touraine A., 1973, Production de la société, Seuil.

Touraine A., 1984, Le Retour de l'acteur, Fayard.

Weber M., 1922/1971, Essais sur la théorie de la science, Plon. 